\title{
Determination Reflection and Transmission Coefficients of Lanthanum Iron Garnet filled PVDF-polymer Nanocomposite using Finite Element Method Modeling at Microwave Frequencies
}

\author{
Hassan Soleimani ${ }^{1, a}$ *, Noorhana Yahya ${ }^{1, b}$, Zulkifly Abbas ${ }^{2, c}$, \\ Hojjatollah Soleimani ${ }^{2, \mathrm{~d}}$ and Hasnah Mohd Zaid ${ }^{1, \mathrm{e}}$ \\ ${ }^{1}$ Fundamental and Applied Science Department, \\ Universiti Teknologi PETRONAS, 31750 Seri Iskandar, Malaysia, \\ ${ }^{2}$ Department of Physics, Faculty of Science, \\ Universiti Putra Malaysia, 43400 UPM Serdang, Malaysia, Malaysia, \\ ahassan.soleimani@petronas.com.my, ${ }^{\text {b}}$ Noorhana_yahya@petronas.com.my, \\ ‘Za@science.upm.edu.my, dhojjatsoleimani@yahoo.com, ${ }^{\circledR}$ hasnamz@petronas.com.my
}

Keywords: Microwave measurements, RW-90 waveguide; LIG, PVDF, FEM

\begin{abstract}
In our previous work, the lanthanum iron garnet-filled PVDF-polymer nanocomposite has been prepared. The reflection and transmission coefficients of PVDF/LIG were measured using rectangular waveguide in conjunction with a microwave vector network analyzer (VNA) at X-band frequencies $(8 \mathrm{GHz}-12 \mathrm{GHz})$. In this study, the distribution of electric field intensity of PVDF/ LIG which was loaded in rectangular waveguide was simulated based on Finite Element Method (FEM) formulation to show the essential differences of intensity of emitted electrical field. The computations of reflection and transmission coefficients of PVDF/ LIG were determined by using implementation FEM modeling rectangular waveguide. The FEM results were compared with the experimental achievement results using the rectangular waveguide. An excellent agreement between measured and simulated results was obtained based on the values of mean relative errors.
\end{abstract}

\section{Introduction}

The application of nanocomposites in microwave and electronic devices requires the exact knowledge of all parameters of a single wave carrier signal. Determination of reflection and transmission coefficients of garnet ferrites loaded polymer nanocomposites have attracted the interest of many researchers and scientists due to their applications in microwave and electronic devices such as isolators, filters and circulators [1-3].

As a soft ferrite material, lanthanum iron garnet $\left(\mathrm{La}_{3} \mathrm{Fe}_{5} \mathrm{O}_{12}\right)$ has been used in various applications in electronic devices. This is due to its efficient absorption of electromagnetic waves, low saturation flux density, low losses at high frequencies, high resistivity and easy to magnetize and demagnetize. As a result, polymer-based composites filled with ferrite particles, such as cobaltferrite [4], NiZn-ferrite [5], and MnZn-ferrite [6, 7] have attracted considerable attention over the years.

The parameters of a single wave carrier signal such as frequency, phase, amplitude and DC component were determined by a general method based on different samples [8] the relative error of the estimated parameters decreases linearly as the signal-to-noise ratio (SNR) increases. For portable DSP, a simple and precise instantaneous frequency estimation method of single sinusoid signals were conducted based on instrumentation to obtain an analytical formula [9]. A quantized multiple sinusoids signal estimation algorithm was presented [10]. The accuracy of the initial values of iterations has a large influence on the speed of convergence. An iterative process was performed in order to reduce the cost function. 
Many methods have been used for measuring the electromagnetic properties of the materials [11]. In our previous work, the transmission reflection rectangular waveguide technique (T/R) was used to determine the reflection and transmission coefficients of the materials [12].

In this study, COMSOL software $[13,14]$ was used to simulate the rectangular waveguide in $3 \mathrm{D}$ geometry. The model consists of a rectangular waveguide with microwave propagation transition through it. This model applies the RF Module's Port boundary condition for the wave propagation problem. With this boundary condition, the software determines the distribution of the electric field intensity based on the Finite Element Method (FEM) [15-19], the reflection and transmission coefficient as a function of frequency were calculated from electrical field distribution. The attenuation of the PVDF/LIG was calculated in decibel (dB) based on the maximum intensity of electric field in rectangular waveguide where the wave enters and exits from it.

\section{Methodology}

Finite element method. In this paper, FEM was used in order to determine the reflection and transmission coefficients of PVDF/LIG loaded in rectangular waveguide. as shown in Fig. 1 It was assumed that the rectangular waveguide was excited by a dominant $\mathrm{TE}_{10}$ mode from the left and the reflection and transmission coefficients were measured at the reference plane $s_{1}$ and $s_{2}$, respectively. For the purpose of analysis, the rectangular waveguide are divided into three regions: Region I, Region II and Region III. The first and third regions (I and III) were filled with air, while the nanocomposite sample filled up the second region (II).

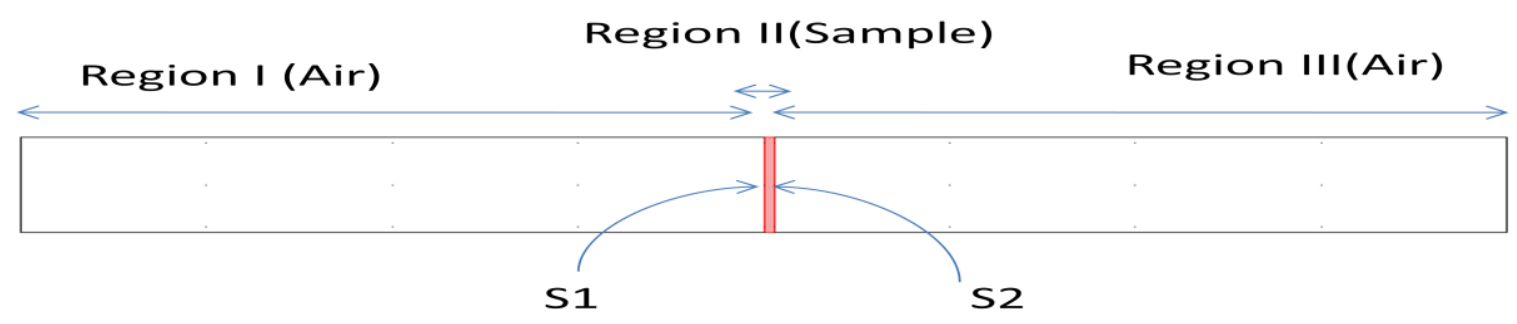

Fig 1: Rectangular Waveguide Loaded with PVDF/LIG Sample.

In the FEM formulation, the electric field in the rectangular waveguide was discretized using tetrahedron elements $[16,17]$ :

$$
\mathrm{E}^{\mathrm{e}}=\sum_{\mathrm{i}=1}^{6} \mathrm{~N}_{\mathrm{i}}^{\mathrm{e}} \mathrm{E}_{\mathrm{i}}^{\mathrm{e}}(\mathrm{x}, \mathrm{y}, \mathrm{z})
$$

where $\mathrm{N}_{\mathrm{i}}^{\mathrm{e}}, \mathrm{i}=1,2,3 \ldots 6$ are the six complex amplitudes of electric field associated with the six edges of the tetrahedron, and $E_{i}^{e}(x, y, z)$ is the vector basis function associated with the $i^{\text {th }}$ edge of the tetrahedron. using boundary condition and integration over the volume of one tetrahedron:

$$
\left[\mathrm{S}_{\mathrm{el}}\right] \times\left\{\mathrm{N}_{\mathrm{i}}^{\mathrm{e}}\right\}=\{\mathrm{v}\}
$$

where $\mathrm{V}$ is found from the boundary condition. These element matrices can be assembled over all the tetrahedron elements in the sample region to obtain a global matrix equation

$$
[\mathrm{S}] \times\left\{\mathrm{N}_{\mathrm{i}}^{\mathrm{e}}\right\}=\{\mathrm{v}\}
$$

The solution vector $\left\{\mathrm{N}_{\mathrm{i}}\right\}$ of matrix Eq. (3) is then used to determine both the reflection and transmission coefficients [18]. 
Sample Preparation. LIG was prepared according to the our previous work [19]. Amorphous LIG was synthesized by the sol-gel method. The pure phase crystalline cubic LIG was obtained by the heat-treatment of the as-prepared amorphous material at $700{ }^{\circ} \mathrm{C}$ for $2 \mathrm{~h}$ in air atmosphere. PVDF/LIG as a nanocomposite sample was prepared by the solvent method with $13 \%$ filler and 87 $\%$ of PVDF in the form of a rectangular sheet with $3 \mathrm{~mm}$ thickness.

Experimental Method. PVDF/LIG as nonocomposite sample was snugly fitted into a WR-90 waveguide then the reflection and transmission coefficients were measured in the frequency range of $8-12 \mathrm{GHz}$ by using an Agilent N5230A PNA-L network analyzer. In this technique, the fundamental transverse electromagnetic (TEM) mode is the only mode that propagates in rectangular waveguide. Network analyzer was calibrated by implementing a standard full two-port calibration technique (SOLT) for 201 frequency points. The experiments were carried out at room temperature.

\section{Results and Discussion}

Electric field distribution. Fig. 2 shows the 2D and 3D plot of FEM simulation rectangular waveguide loaded mentioned nanocomposite sample. General observation shows that the essential differences of emitted electric field intensity in difference regions of rectangular waveguide at 12 $\mathrm{GHz}$.
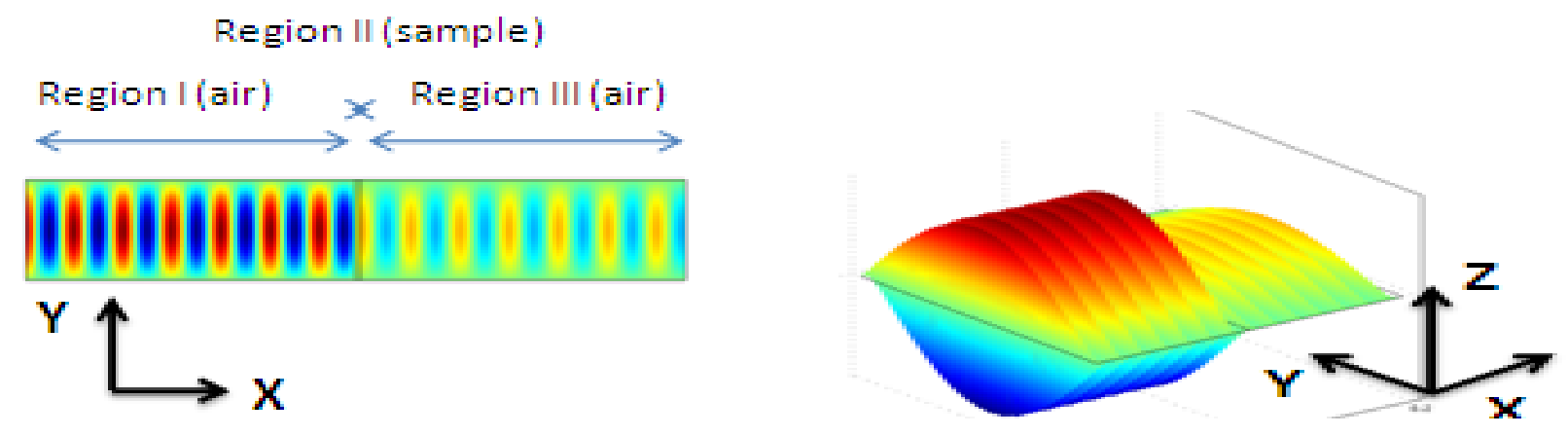

Fig 2: FEM Simulation of Electric Intensity for $3 \mathrm{~mm}$ thick of PVDF/YIG in 2D and 3D Surface Plots.

Fig. 3 shows the variation of z-component of the electrical field versus the length of the rectangular waveguide. The $\mathrm{x}$ - axis represents the length of a rectangular waveguide from -0.2 to $0.2 \mathrm{~m}$, and the $\mathrm{z}$ axis indicates the $\mathrm{z}$ component of the electric field.

In region I, the maximum electric field amplitude presented was as high as $405 \mathrm{~V} / \mathrm{m}$ ). When the wave was propagated through the sample, the electric field amplitude decreased to $144 \mathrm{~V} / \mathrm{m}$, only $0.275 \%$ of one wavelength of propagated wave is in region II due to $3 \mathrm{~mm}$ thickness of sample. This result is expected where the wavelength of propagated wave in PVDF/LIG at $12 \mathrm{GHz}$ frequency has a value of $10.86 \mathrm{~mm}$ as the refractive index has value 2.30 as well which is validated by FEM simulation results. Fig. 3 shows that the electrical field amplitude increased to $146 \mathrm{~V} / \mathrm{m}$ where the wave exiting from the region II to the region III of rectangular waveguide. Therefore, the attenuation of a $3 \mathrm{~mm}$ thick of PVDF/LIG at $12 \mathrm{GHz}$ frequency was achieved at $9.30 \mathrm{~dB}$ due to the maximum intensity of electric fields in entrance region I and exit from region III at rectangular waveguide. 


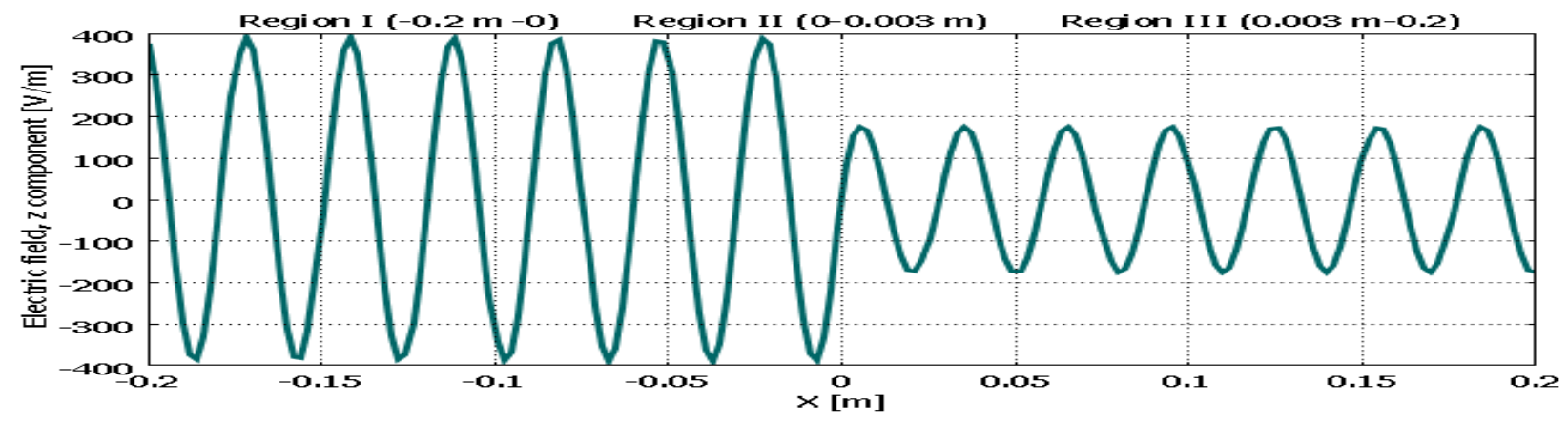

Fig 3: Variation of the Z Component of Electric Field with respect to the Length of Rectangular Waveguide in Region I, II, and III.

FEM simulation of the rectangular waveguide loaded with $3 \mathrm{~mm}$ thick of PVDF/LIG and rectangular waveguide free of sample were conducted at a different number of elements. The relationship between the number of tetrahedron elements (meshes) and attenuation at $12 \mathrm{GHz}$ for both mentioned rectangular waveguide is shown in Fig. 4. The results indicate that the attenuations are irregular and mistake when the element factor (number of mess) is less than 3500. Increasing the number of meshes from 3500 to higher, the results became more regular and accurate. In rectangular waveguide loaded with PVDF/LIG, the attenuation increase with an increase of the number of meshes. while the values of attenuation in rectangular waveguide free of sample is shown to be constant.

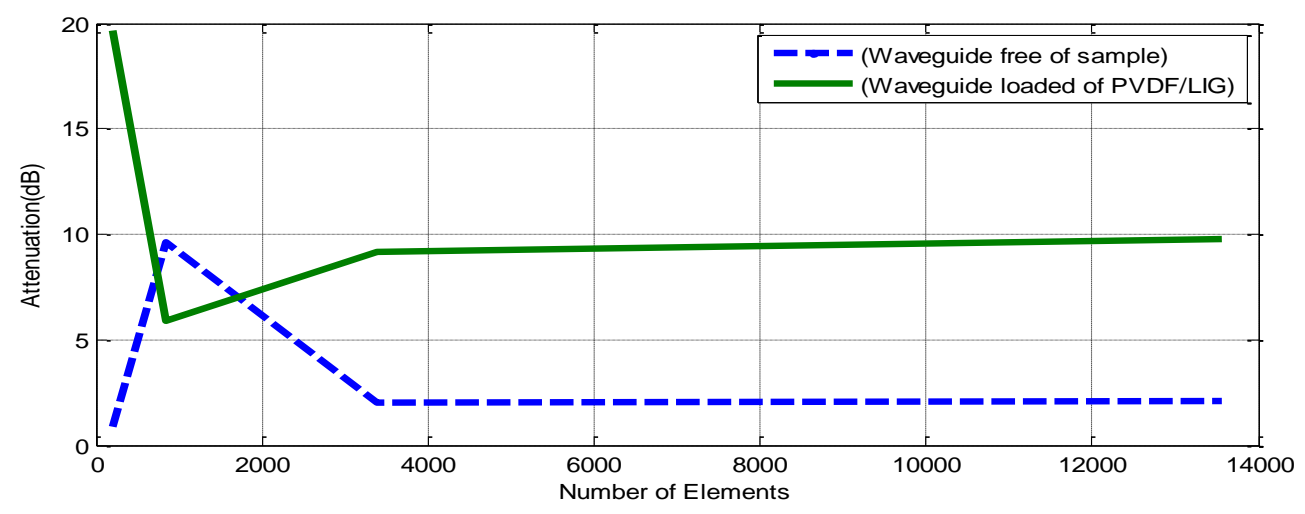

Fig 4: The Relationship between Number of Mesh and Attenuation at $12 \mathrm{GHz}$ for PVDF/LIG and Free space rectangular waveguide.

Reflection and Transmission Coefficient. Fig. 5 ( $a$ and b) shows the comparisons between the FEM simulation and measured data for variation in reflection and transmission coefficients. PVDF/LIG fitted to the rectangular waveguide, and the relative permittivity and permeability values were $\varepsilon_{\mathrm{r}}=4.33-\mathrm{j} 0.09, \mu_{\mathrm{r}}=1.24-\mathrm{j} 0.15$ respectively [20]. As shown in Fig. 5, the $\mathrm{X}$-axis represents the frequencies from 8 to $12 \mathrm{GHz}$ and the Y-axis indicates a tolerance from zero to one.

General observation shows that the level of transmission is greater than reflection. The sum of reflection and transmission coefficient values is always around unity. Hence, the increase in reflection coefficient causes the decrease of transmission coefficient and vice versa. As can be seen in Fig. 5, the measured and simulated (FEM) values for reflection coefficient start at 0.54 and 0.64 respectively from the $\mathrm{Y}$ axis. From this point, the trend of the measured and simulated curves of reflection coefficient demonstrates a decreasing to 0.52 and 0.53 when the frequency reaches 12 GHz. In contrast, the measured and simulated (FEM) values in transmission coefficient start at 0.70 and 0.64 respectively from the $\mathrm{Y}$ axis. From this point, the trend of the measured and simulated curves remains constant when the frequency reaches $12 \mathrm{GHz}$ in the $\mathrm{X}$ axis. 


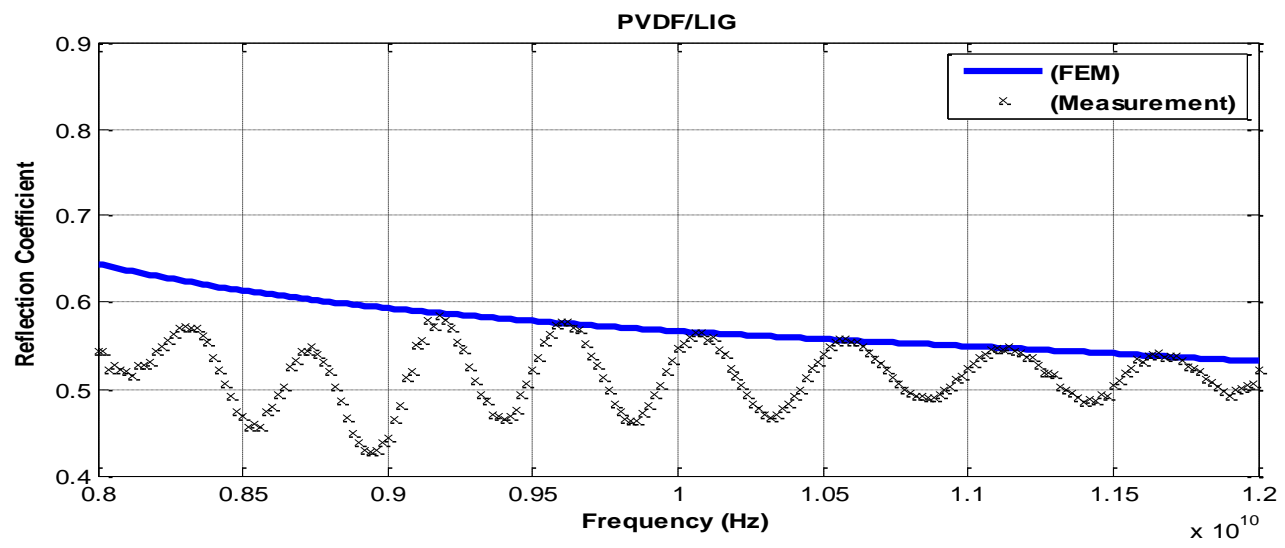

(a)

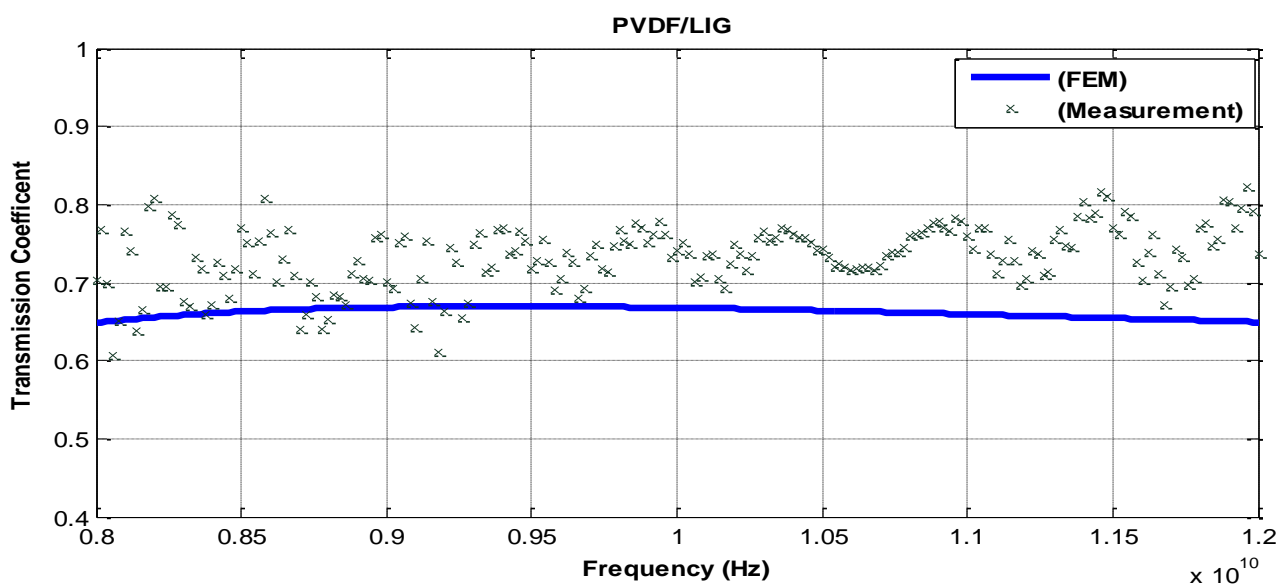

(b)

Fig 5: Measured and Simulated Magnitude of (a) Reflection Coefficient and (b) Transmission Coefficient of a $3 \mathrm{~mm}$ thick of PVDF/LIG at X-band Frequencies.

The accuracy of FEM simulation of reflection and transmission coefficients can be determined by calculating the relative error with respect to the measurement data. The mean relative error values of FEM simulation of the reflection and transmission coefficients was observed to be 0.0596 and 0.0972, respectively. Consequently, the FEM simulation results in reflection coefficient are more accurate than transmission coefficient where PVDF/LIG as nanocomposite sample examined.

\section{Conclusion}

In this paper, a 3D Finite Element Modeling procedure was presented to determine the reflection and transmission coefficients of PVDF/LIG loaded in rectangular waveguide. The results were in good agreement with the result by measured data found using vector network analyzer as the mean relative error values of FEM simulation of reflection and transmission coefficients observed to 0.0596 and 0.0972 , respectively. The simulation of distribution electric field intensity in rectangular waveguide was conducted. Therefore, it indicated that the attenuation of a $3 \mathrm{~mm}$ thick of PVDF/LIG at $12 \mathrm{GHz}$ frequency revealed to $9.30 \mathrm{~dB}$. It was due to the maximum electric field amplitudes as high as 405 and $146 \mathrm{~V} / \mathrm{m}$ at regions I and III, respectively in rectangular waveguide. 


\section{References}

[1] P. Toneguzzo, O. Acher, G. Viau, F. Fievet-Vincent, F. Fievet, Observations of exchange resonance modes on submicrometer sized ferromagnetic particles, J. Appl. Phys. 81 (1997) $5546-5548$.

[2] M. Pardavi-Horvath, Characterization of nanostructured magnetic materials, J. Magn. Magn. Mater. 203 (1999) 57-59.

[3] Y.J. Wu, H.P. Fu, R.Y. Hong, Y. Zheng, D.G. Wei, Influence of surfactants on coprecipitation synthesis of Bi-YIG particles, J. Alloys Compd. 470 (2009) 497-501.

[4] P.P. Vaishnava, U. Senaratne, E. Buc, R. Naik, V.M. Naik, G. Tsoi, L.E. Wenger, P. Boolchand, Magnetic properties of cobalt-ferrite nanoparticles embedded in polystyrene resin, J. Appl. Phys. 99 (2006) 702-703.

[5] T. Nakamura, T. Tsutaoka, K. Hatakeyama, Frequency dispersion of permeability in ferrite composite, J. Magn. Magn. Mater. 138 (1994) 319-328.

[6] O. Yavuz, M.K. Ram, M. Aldissi, P. Poddar, S. Hariharan, Synthesis and the physical properties of MnZn ferrite and NiMnZn ferrite-polyaniline nanocomposite particles, J. Mater. Chem. 15 (2005) 810-817.

[7] N.E. Kazantseva, Y.I. Bespyatykh, I. Sapurina, J. Stejskal, J. Vilcáková, P. Sáha, Magnetic materials based on manganese-zinc ferrite with surface-organized polyaniline coating, J. Magn. Magn. Mater. 301 (2006) 155-165.

[8] D.N Vizireanu, S.V. Halunga, Single sine wave parameters estimation method based on four equally spaced samples, Int. J. Electronics 98(2011) 941-948.

[9] D.N Vizireanu, A simple and precise real-time four point single sinusoid signals instantaneous frequency estimation method for portable DSP based instrumentation Measurement , J.Measurement 44( 2011) 500-502.

[10] J. Baker, Dielectric and magnetic measurement methods in transmission lines: an overview, Proceedings of the AMTA Workshop, Chicago, Illinios. 1992.

[11] S. Biju Kumar, H. Hohn, R. Joseph, M. Hajian, L.P. Ligthart, K.T. Mathewa, Complex permittivity and conductivity of poly aniline at microwave frequencies, J. European Ceramic Society 21 (2001) 2677-2680.

[12] H. Soleimani, Z. Abbas, K. Khalid, N. Yahya and H. Soleimani, Determination SParameters of PTFE at X-band Frequency using FEM Modeling Rectangular Waveguide, European Journal of Scientific Research 39(2010) 105-110.

[13] The Comsol Multiphysics, Quick start and Quick Reference, version 3.3 Comsol AB, 2006.

[14] M. N. O. Sadiku, Elements of electromagnetic, Oxford University Press, New York, 2001.

[15] P. P. Silvester, R. L. Ferrari, Finite Elements for Electrical Engineers, Second ed., Cambridge Univ. Press, 1990. 
[16] C. J. Reddy, M. D. Deshpande, C.R. Cockrell, F.B. Beck, Finite element method for eigenvalue problems in electromagnetic, NASA Technical Paper 3485(1994).

[17] C. J. Reddy, M. D. Deshpande, Application of FEM to estimate complex permittivity of dielectric material at microwave frequency using waveguide measurements, NASA Contractor Report 1995.

[18] R. Coccioli, G. Pelosi, S. Selleri, Characterization of Dielectric Materials with the FiniteElement Method, IEEE Trans. Microw. Theo. Tech. 47(1999).

[19] H. Soleimani, Z. Abbas, N. Yahya, H. Soleimani and M. Y. Ghotbi, Microwave characterization of lanthanum iron garnet-filled PVDF-polymer composite using rectangular waveguide at X-band frequency, J. Composite Materials 46 (2011) 1497-1501.

[20] H. Soleimani, Z. Abbas, N. Yahya, H. Soleimani and M.Y. Ghotbi, Determination of Complex Permittivity and Permeability of lanthanum iron garnet filled PVDF-polymer composite Using rectangular Waveguide and Nicholson-Ross-Weir (NRW) Method at Xband frequencies, J. Measurement 45(2012)1621-1625. 